\title{
Successful Spinal Cord Stimulator
}

Received: 2019-04-08

Accepted: 2019-07-24

Trial Placement in a Patient

Published: 2020-03-30

\section{with Presumed Heparin-Induced \\ Thrombocytopenia Treated With \\ Argatroban: A Case Report}

\author{
Mario Flores, DO 1 \\ Elizabeth M. Leimer, MD, PhD² \\ Scott Chamberlain, $\mathrm{DO}^{1}$ \\ Yousaf Chowdhry, MD' \\ Andras Laufer, MD' \\ Ravneet Bhullar, MD
}

Background: Interventional pain procedures such as spinal cord stimulator placement are safely performed when anticoagulation medications are discontinued beforehand in accordance with published recommendations. However, current guidelines for direct thrombin inhibitors are limited to dabigatran, rivaroxaban, apixaban, and edoxaban. One recommendation is to allow a 5-half-life interval between discontinuation of these medications and a high-risk interventional spine procedure to avoid complications such as spinal hematoma.

Case Report: We report a case of a 53-year-old woman with multiple comorbidities who was placed on a heparin infusion after presenting with acute radial artery occlusion and right hand ischemia. The patient underwent vascular bypass of the right arm and then developed compartment syndrome postoperatively, which was treated via fasciotomy. The patient subsequently developed heparin-induced thrombocytopenia and began argatroban for anticoagulation. The patient developed severe right upper extremity ischemic pain, but both medical management and treatment via peripheral nerve catheters failed to control her pain. A cervical spinal cord stimulator trial was placed. The patient did not report significant pain relief after 7 days, so the spinal cord stimulator was removed. Five half-lives were used for discontinuation of argatroban before both spinal cord stimulator trial lead placement and removal. Five half-lives were also used for restarting argatroban following these procedures. No complications were seen with the placement or removal of spinal cord stimulator leads.

Conclusion: This case report demonstrates that discontinuing argatroban 5 half-lives before cervical spinal cord stimulator trial placement can be done safely in this patient population and reveals the need for larger case studies to provide additional evidence for guideline recommendations.

Key words: Anticoagulation guidelines, direct thrombin inhibitors, interventional pain, interventional spine, ischemic pain, neuromodulation

1 Albany Medical Center, Department of Anesthesiology, Albany, NY

2 Albany Medical College, Albany, NY

Corresponding Author: Ravneet Bhullar, MD, Albany Medical Center, Department of Anesthesiology, 47 New Scotland Ave, MC 131, Albany, NY 12208,

E-mail: bhullar@amc.edu

Conflict of Interest: None Declared

Disclaimer: There was no external funding in the preparation of this manuscript. Conflict of interest: Each author certifies that he or she, or a member of his or her immediate family, has no commercial association (i.e., consultancies, stock ownership, equity interest, patent/licensing arrangements, etc.) that

might pose a conflict of interest in connection with the submitted manuscript. 


\section{BACKGROUND}

Interventional pain procedures such as spinal cord stimulator (SCS) placement are safely performed when anticoagulation medications are discontinued before the procedure in accordance with published guidelines $(1,2)$. Recommendations for patients needing a highrisk pain procedure while on direct thrombin inhibitors exist $(1,3)$, but are limited to dabigatran, rivaroxaban, apixaban, and edoxaban. There are no current guidelines regarding the use of argatroban in the context of a high-risk interventional pain procedure such as SCS placement.

An interest in argatroban began when a patient at Albany Medical Center developed heparin-induced thrombocytopenia (HIT) while on a heparin infusion for treatment of peripheral thrombus and limb ischemia (4). Argatroban was instituted for anticoagulation, which is a direct thrombin inhibitor indicated for treatment of thrombosis associated with HIT (4). Argatroban has a reported terminal elimination half-life of 39 to 51 minutes and is metabolized by hydroxylation and aromatization of the 3-methyltetrahydroquinoline ring in the liver (5). Total body clearance is approximately $5.1 \mathrm{~mL} / \mathrm{kg} / \mathrm{min}(0.31 \mathrm{~L} / \mathrm{kg} / \mathrm{hr})$ for infusion doses up to 40 $\mathrm{mcg} / \mathrm{kg} / \mathrm{min}$ (5). Hepatic impairment is associated with decreased clearance and increased elimination half-life of argatroban. No metabolic effects are reported with renal impairment (5). While on argatroban, the patient developed severe ischemic limb pain, and SCS placement was considered after failure of other pain management modalities.

Planning SCS placement in this patient required careful assessment of the American Society of Regional Anesthesia (ASRA) and American Society of Interventional Pain Physicians (ASIPP) guidelines and literature for antiplatelet and anticoagulation medication for high-risk interventional spine procedures $(1-3,6)$, as well as other comorbidities to avoid complications such as spinal hematoma. Spinal hematomas occur at an incidence of one in 150,000 while on anticoagulation (7). The incidence of epidural hematoma with SCS placement while on argatroban anticoagulation is unknown, as argatroban has been avoided with high-risk procedures. The current ASRA recommendations for patients undergoing a medium- or high-risk interventional spine procedure and on direct thrombin inhibitors (dabigatran, rivaroxaban, apixaban, edoxaban) include (1):

- A 5-half-life interval between discontinuation of direct thrombin inhibitor and the procedure;
- Bridging with low-molecular-weight heparin (LMWH) if the risk of venous thromboembolism (VTE) is high, and discontinuing LMWH 24 hours prior to the procedure;

- Giving half of the usual dose 12 hours prior to the procedure if the risk of VTE is very high and there is physician consensus;

- Restarting the direct thrombin inhibitor 24 hours after the procedure.

Prior literature describes spinal hematomas following SCS placement as a complication associated with either aspirin therapy $(8,9)$, or did not consider anticoagulation or antiplatelet therapy when analyzing outcomes $(10,11)$. One case report demonstrated successful permanent SCS implantation for a patient on chronic dual antiplatelet therapy with aspirin and clopidogrel (12). Multiple case reports have described an association between rivaroxaban, a direct thrombin inhibitor, and spinal hematoma $(13,14)$. However, no literature exists that describes high-risk interventional spine procedures or spinal anesthesia while on argatroban.

This case report is the first to describe SCS placement in a patient on argatroban. Similar to the current ASRA guidelines for other direct thrombin inhibitors, discontinuing argatroban 5 half-lives before SCS placement may be safe for patients with a history of multiple thromboemboli and HIT.

\section{METHODS}

Institutional Review Board (IRB) approval was not needed for the purposes of this case report at our institution. The patient described in this case study consented to the use of the case in our report.

The patient, a 53-year-old woman, was initially admitted with right hand pain and ischemia and found to have an occlusive thrombus in both the radial and ulnar arteries. Her most significant comorbidities were coronary artery disease with a prior myocardial infarction and remote right clavicle fracture with fixation. She initially underwent right ulnar-to-ulnar vascular bypass surgery with reverse saphenous vein graft and was started on a heparin infusion. Postoperatively she developed compartment syndrome in her right extremity and required emergent fasciotomies and revision of the right arm bypass. On hospital day 18 , she developed thrombocytopenia due to presumed HIT with positive platelet factor 4 (PF4). She was evaluated by the hematology service and anticoagulation was changed from heparin infusion to argatroban. The subsequent serotonin release assay was negative, and although this 
result made true HIT unlikely, argatroban was continued in the setting of thrombocytopenia with positive PF4, optical density 1.9, and high 4T score.

The acute pain service was consulted on hospital day 3. Her pain was located in the right extremity, but was worse in the right fingers and described as continuous and burning in nature. She had peripheral nerve catheters for 7 days with good sensory levels, but these failed to relieve the burning pain in her fingers despite receiving $200 \mathrm{mg}$ of celecoxib twice per day, $60 \mathrm{mg}$ of duloxetine daily, gabapentin $900 \mathrm{mg} 3$ times per day, $40 \mathrm{mg}$ of oxycodone twice per day, $8 \mathrm{mg}$ of tizanidine 3 times per day, $400 \mathrm{mg}$ of magnesium oxide twice per day, hydromorphone patient-controlled analgesia, and oxycodone and acetaminophen as needed.

After various interventions failed to control her pain, she was offered a cervical SCS trial which was placed on hospital day 37. ASRA and ASIPP recommendations for dabigatran use (half-life of 12 to 17 hours) in medium- to high-risk pain procedures include cessation of the drug 5 half-lives before the procedure and resumption 24 hours after the procedure $(1,6)$. Due to the lack of guidelines on cessation and resumption of argatroban with this procedure, 5 pharmacological half-lives were used based on these recommendations. This was determined to be the most conservative approach, as after 5 half-lives only $3.125 \%$ of the drug remains in the patient, versus $25 \%$ which remains after 2 half-lives. Beyond 5 half-lives, the risk-benefit ratio changes. The risk of a thromboembolic event may increase due to the increased likelihood of developing a deep vein thrombosis. Given the highrisk nature of the procedure, the anatomic location, and the patient being on a serotonin-norepinephrine reuptake inhibitor (SNRI), we chose to use 5 half-lives. Argatroban was stopped 4 hours before placement. No other anticoagulants were used before or after the procedure. At this time, her platelets were 303, international normalised ratio (INR) 1.1, prothrombin time (PT) 11.7, partial thromboplastin time (PTT) 42. The patient was positioned prone for the procedure and communicated with the team throughout the case. With fluoroscopic guidance, a 14-gauge Tuohy needle was advanced to contact the right T3 lamina and then advanced superomedially until it entered the epidural space, checked using the loss-of-resistance technique with air. The leads ( $250-\mathrm{cm}$ InfinionTM 16 contact trial leads) were advanced though the Tuohy needle and directed to the tip of the $\mathrm{C} 2$ vertebral body on the left side. The procedure was repeated for the right side with the lead on the right adjacent to the left lead. The procedure was uncomplicated and the patient tolerated the procedure well. The patient restarted argatroban 4 hours after the procedure. She failed to have any pain relief with 48 hours of spinal cord stimulation. After a 6-day trial, argatroban was held for 4 hours and the leads were removed easily, atraumatically, without any resistance, and with both visibly intact. The patient was restarted on argatroban 4 hours after removal. Her pain was medically managed for the remainder of her hospital stay and she was seen in the outpatient clinic following discharge.

\section{RESULTS}

No complications were observed with either SCS lead placement or removal when argatroban was discontinued 5 pharmacological half-lives before the procedure.

\section{DISCUSSION}

ASRA and ASIPP have recently published recommendations for interventional spine procedures and direct thrombin inhibitors $(1,6)$, but there are no similar guidelines regarding timing schedules for cessation and resumption of argatroban in this context.

As noted in ASRA guidelines, 2 to 5 pharmacological half-lives can be used when deciding timing of cessation and resumption of an anticoagulant medication (1). In our case, we elected to be more conservative given the high-risk nature of the procedure. SCS lead placement has been categorized as high-risk due to the use of high-gauge needles, multiple insertions in the epidural space, and manipulation of leads all potentially increasing the risk of trauma to the epidural space (1). Placing leads in the cervical epidural space is also high-risk, as the epidural space is smaller than that of the thoracic and lumbar spine (C7-T1: $0.4 \mathrm{~mm}$, thoracic and lumbar regions: 4-7 $\mathrm{mm}$ ) (15). However, the volume of the epidural space was not determined prior to SCS placement, as cervical spine magnetic resonance imaging (MRI) was not obtained. Therefore, the size of the epidural space compared to the lead dimensions and the resulting likelihood of increased trauma due to shear forces is unknown. Additionally, SNRIs have been shown to affect clotting and increase the risk of bleeding (1) and this patient was taking duloxetine at the time of the procedure. Taken together with her additional risk factors, we chose to use the most conservative approach for anticoagulant management.

While prevention of spinal hematoma is of paramount importance, cessation of anticoagulation can put the patient at risk for a thromboembolic event (3). Given the 
hypercoagulable state of the patient and the high-risk nature of the procedure, 5 pharmacological half-lives were chosen due to the relatively short half-life of argatroban.

This case report shows that a high-risk interventional spine procedure such as cervical SCS placement can be done safely while a patient is on argatroban, but larger case series studies are needed to provide suitable evidence for guideline recommendations.

\section{REFERENCES}

1. Narouze S, Benzon HT, Provenzano D, et al. Interventional spine and pain procedures in patients on antiplatelet and anticoagulant medications (second edition): Guidelines from the American Society of Regional Anesthesia and Pain Medicine, the European Society of Regional Anaesthesia and Pain Therapy, the American Academy of Pain Medicine, the International Neuromodulation Society, the North American Neuromodulation Society, and the World Institute of Pain. Reg Anesth Pain Med 2018; 43:225-262.

2. Kaye AD, Trang TN, Cornett EM, Ehrhardt KP, Shah RV. Anticoagulation and regional anesthesia concerns. In: Kaye A, Urman R, Vadivelu N (eds). Essentials of Regional Anesthesia. Springer, Cham, 2018, pp. 121-134.

3. Horlocker TT, Vandermeuelen E, Kopp SL, Gogarten W, Leffert LR, Benzon HT. Regional anesthesia in the patient receiving antithrombotic or thrombolytic therapy: American Society of Regional Anesthesia and Pain Medicine Evidence-Based Guidelines. Reg Anesth Pain Med 2018; 43:263-309.

4. Bambrah RK, Pham DC, Rana F. Argatroban in heparin-induced thrombocytopenia: Rationale for use and place in therapy. Ther Ad Chronic Dis 2013; 4:302-304.

5. Argatroban Full Prescribing Information: Clinical Pharmacology. North Wales, PA: Teva Pharmaceuticals USA, Inc; August 2017. Reference ID: 4143729.

6. Kaye AD, Manchikanti L, Novitch MB, et al. Responsible, safe, and effective use of antithrombotics and anticoagulants in patients undergoing interventional techniques: American Society of Interventional Pain Physicians (ASIPP) Guidelines. Pain Physician 2019; 22:S1-S74.

\section{CONCLUSION}

The management of less common anticoagulant agents during an interventional procedure can be complicated due to limited available data and few published guidelines. This case report provides an example of successful and appropriate management of SCS lead placement in a patient on argatroban for anticoagulation.

7. Tryba M. Epidural regional anesthesia and low molecular heparin [in German]. Anasthesiol Intensivmed Notfallmed Schmerzther 1993; 28:179Y181.

8. Buvanendran A, Young AC. Spinal epidural hematoma after spinal cord stimulator trial lead placement in a patient taking aspirin. Reg Anesth Pain Med 2014; 39:70-72.

9. Giberson CE, Barbosa J, Brooks ES, et al. Epidural hematomas after removal of percutaneous spinal cord stimulator trial leads: Two case reports. Reg Anesth Pain Med 2014; 39:73-77.

10. Spincemaille GH, Klomp HM, Steyerberg EW, van Urk H, Habbema JD. Technical data and complications of spinal cord stimulation: Data from a randomized trial on critical limb ischemia. Stereotact Funct Neurosurg 2000; 74:63-72.

11. Chen $H$, Yuan A. Delayed onset epidural hematoma after permanent spinal cord stimulator implant. J Pain 2015; 16:S70.

12. Covert BP, Nobles RH. Successful spinal cord stimulator trial and permanent implant in patient with diabetic peripheral neuropathy on chronic dual antiplatelet therapy. Pain Physician 2015; 18:E905-E909.

13. Madhisetti KR, Mathew M, George M, Pillai SS. Spinal epidural haematoma following rivaroxaban administration after total knee replacement. Indian J Anaesth 2015; 59:519-521.

14. Zaarour M, Hassan S, Thumallapally N, Dai Q. Rivaroxaban-induced nontraumatic spinal subdural hematoma: An uncommon yet lifethreatening complication. Case Rep Hematol 2015; 2015:275380.

15. Nickalls RW, Kokri MS. The width of the posterior epidural space in obstetric patients. Anaesthesia 1986; 41:432-433. 